\title{
Tunable rectangular ring resonator filter with embedded shunt barium strontium titanate capacitors
}

\begin{abstract}
Design of a low loss tunable bandpass filter using a microstrip ring resonator is presented. The tunability is achieved through the use of embedded shunt parallel plate variable capacitor implemented into the Low Temperature Cofired Ceramic (LTCC) substrate using thick film Barium Strontium Titanate (BST) paste. Measured data on the filter response, loss, and tunability in the frequency of $10 \mathrm{GHz}$ and $12 \mathrm{GHz}$ is presented. Results show filters produced low insertion loss with narrow tenability range.
\end{abstract}

Keyword: Resonator filter; Tunable filter; Ferroelectric capacitors 